\title{
Individual Particles, Properties and Quantum Statistics
}

\begin{abstract}
Although there have been several attempts to resist this conclusion, it is commonly held that the peculiar statistical behaviour of quantum particles is due to their nonindividuality. In this paper, a new suggestion is put forward: quantum particles are individuals, and the distinctive features of quantum statistics are determined by the fact that all the state-dependent properties described by quantum statistics are emergent relations.
\end{abstract}

\section{Introduction}

A long-standing debate in the philosophy of quantum mechanics concerns whether or not particles are - or at least can consistently be said to be - individuals, that is, entities that are determinately self-identical and numerically distinct from other entities.

Those who favour an affirmative answer - perhaps in view of the fact that it seems to minimise the 'gap' between our everyday experience of physical entities and what our best science tells us about the ultimate basis of such an experience must cope with two difficulties:

- The fact that the Principle of the Identity of the Indiscernibles is violated in quantum mechanics, but is considered by many a plausible criterion on the basis of which to attribute individuality to things;

- The 'non-classicality' of quantum statistics, whose peculiarities are readily explained by assuming that quantum systems are composed of non-individuals. ${ }^{1}$

\footnotetext{
${ }^{1}$ The claim that since quantum particles obey a non-classical statistics they should be regarded as non-individuals can be found as early as Born [1926]. This position has later become the 'Received View' on the nature of quantum entities (see, for instance, the historical reconstruction in French and Krause [2006; Chapter 3]).
} 
The former problem can only be overcome by postulating some form of primitive thisness that individuates the particles independently of their qualities. ${ }^{2}$ This paper assumes that this is reasonable, and deals with the latter difficulty. Section 1 gives an outline of classical and quantum statistics, explaining why the latter is commonly taken to entail non-individuality. Section 2 considers some existing attempts to avoid this conclusion and their problems. Section 3 looks at the account according to which quantum particles are individuals with state-accessibility restrictions, and explains the trouble with surplus structure that such an account meets with. Section 4 puts forward an alternative proposal, based on a form of 'ontological revision': namely, on the idea that all many-particle quantum systems exclusively exhibit (state-independent properties and properties of the total system having been set aside) emergent relations that are entirely independent of the identities of their relata (i.e., of the particles). Section 5 adds a few specifications; in particular, a required modification to the established interpretation of quantum mechanics is made explicit. A concluding section follows.

\section{Classical and Quantum Statistics}

An outline of the basic features of quantum statistics is sufficient for understanding what the issue discussed here amounts to.

Suppose one has $\mathrm{N}$ particles distributed over $\mathrm{M}$ possible single-particle microstates, and is interested in knowing the number of physically possible combinations.

In classical mechanics, Maxwell-Boltzmann statistics holds. According to it, the number of possible distributions is

$\mathrm{W}=\mathrm{M}^{\mathrm{N}}$

In the case of quantum particles, fewer arrangements are available. Bose-Einstein statistics (which applies to the particles known as bosons) has it that

$\mathrm{W}=(\mathrm{N}+\mathrm{M}-1)$ ! /N! (M-1)!

In the case of fermions, the Exclusion Principle (dictating that no two fermions can be in the same state) holds and further reduces the number of possible states, which becomes equal to

\footnotetext{
${ }^{2}$ See, for a classic statement of this view, French and Redhead [1988]. This holds at least for bosons: see Saunders [2006] for the suggestion that fermions are individuated qualitatively by irreflexive relations. (This suggestion appears problematic, but there is no space to discuss it here).
} 
$\mathrm{W}=\mathrm{M} ! / \mathrm{N} !(\mathrm{M}-\mathrm{N})$ !

The latter expresses so-called Fermi-Dirac statistics. ${ }^{3}$

On the basis of the above, one can calculate the probability of a specific configuration being realised. This is, obviously enough, given by

$\operatorname{Prob}(s)=\mathrm{T} / \mathrm{W}$

with $s$ being the arrangement in question, and $\mathrm{T}$ the number of ways in which $s$ can be actualised.

For example, consider a physical system composed of two individuals to each one of which two equally probable states are available (that is, for which $\mathrm{M}=\mathrm{N}=2$ ). Think, for example, of a system composed of two fair coins. Classically, one applies $\mathrm{MB}$ and obtains $2^{2}=4$ possible arrangements, each one with probability $1 / 4$ of being realised. In quantum mechanics, instead, there are only either $(2+2-1) ! / 2 !(2-1) !=3$ possibilities $(\mathrm{BE})$ or 2 ! $/ 2$ ! (2-2)! $=1$ possibility (FD). The probabilities are $1 / 3$ and 1 , respectively. Given this, it can be easily seen that, for instance, in the classical case the probability of an arrangement with the coins in different states is $1 / 2$, while in the quantum case it is either $1 / 3(\mathrm{BE})$ or $1(\mathrm{FD})$.

In more detail, the arrangements available in the situation being considered are the following ( $x$ and $y$ being the available states, and with the subscripts taken to denote the - alleged particle identities):

$$
\begin{aligned}
& \left|x>_{1}\right| x>_{2} \\
& \left|y>_{1}\right| y>_{2} \\
& \left|x>_{1}\right| y>_{2} \\
& \left|y>_{1}\right| x>_{2} \\
& 1 / \sqrt{2}\left(\left|x>_{1}\right| y>_{2}+\left|y>_{1}\right| x>_{2}\right) \\
& 1 / \sqrt{2}\left(\left|x>_{1}\right| y>_{2}-\left|y>_{1}\right| x>_{2}\right)
\end{aligned}
$$

$\mathrm{C} 1-\mathrm{C} 4$ are possible particle arrangements in classical mechanics, Q1-Q4 the configurations available in quantum mechanics. In particular, Q1, Q2 and Q3 are symmetric states, accessible for bosons; and Q4 the unique possible state for fermions (which is anti-symmetric). ${ }^{4}$

\footnotetext{
${ }^{3}$ This tri-partition may be disputed, for example on the basis of the claim that classical systems obeying Bose-Einstein statistics are both theoretically and practically possible (see Gottesman [2005]). However, it looks as though a general distinction can in fact be drawn meaningfully on the basis of what is the case under normal circumstances.

${ }^{4} \mathrm{Q} 3$ and Q4 describe the entangled states typical of quantum mechanics.
} 
Thus, in quantum mechanics only (anti-)symmetric states are possible. ${ }^{5}$ For such states, particle exchanges do not make a difference: simply put, there is only one way for two 'quantum coins' to be one heads and one tails (quantum particles are said to be indistinguishable).

Moreover, non-symmetric states are impossible in the quantum case (that is, analogues of C3 and C4 for quantum particles are never realised). This appears particularly problematic for those who want to defend the particles' individuality. If particles truly are individuals, the question is, why are states that would differ from allowed ones (Q1 and Q2 above) only as regards which value is exhibited by which particle not possible? ${ }^{6}$

The available evidence, the canonical argument goes, suffices to conclude in favour of the non-individuality of quantum particles. First, particle permutations cannot in principle make a difference in the quantum case because quantum particles are not individuals, and so there are no identities that can be permuted. Similarly, non-symmetric states are ruled out because in the quantum domain it is impossible for a specific particle to have a certain value for an observable and for another specific one to have a different value for that observable, as the particles do not have determinate identities allowing for such property-attributions.

\section{Attempts to Avoid the Conclusion}

Faced with the above evidence, one who does not like the idea that quantum particles are not individuals can do two things. One possibility is to show that - contrary to what is commonly believed - classical particles do not in fact possess those distinctive features, allegedly essential to individuals, which are lacking in the quantum domain. The other alternative is more directly to resist the conclusion drawn in the quantum case.

One well-known argument going in the former direction is grounded on the idea that classical particles are in fact as

\footnotetext{
${ }^{5}$ Also, the two types of quantum particles necessarily conserve their symmetry-type, i.e., there are no transitions from a fermionic to a bosonic state (or vice versa): this is known as the Symmetrisation Postulate.

${ }^{6}$ As will be seen in what follows, this question rests on the tacit assumption that there is a property possessed by each particle as its property.
} 
indistinguishable as quantum particles and, therefore, indistinguishability is insufficient for non-individuality. ${ }^{7}$ This view is motivated by the fact that Maxwell-Boltzmann statistics incorrectly predicts that mixing similar gases at the same pressure and temperature one experiences a change in entropy (this is the so-called 'Gibbs' paradox'). Since, as a consequence of this, one has to introduce an N! factor excluding permutations in order to make entropy correctly extensive, it might be concluded that classical many-particle systems are permutation invariant too.

However, it can be immediately responded that, historically, the failure of extensiveness for entropy in the context of classical statistics is exactly what led to the shift to quantum mechanics. That is, that by eliminating permutations one is in fact switching to a non-classical setting. ${ }^{8}$ Following the avenue of classical indistinguishability, therefore, does not seem to be a very promising strategy for the supporter of individuality in quantum mechanics.

A more complex plan, aiming to deny the existence of any metaphysical difference between classical and quantum mechanics, is developed by Huggett in a series of works ([1995], [1997] and [1999]). Huggett argues that the idea that particle permutations should make a difference if particles are individuals depends on a supposition as to the truth of haecceitism: namely, the metaphysical doctrine according to which possible worlds can differ exclusively with respect to the identities of the entities inhabiting them (i.e., be distinct worlds in spite of the fact that they are qualitatively identical). However, Huggett argues, haecceitism is by no means necessary for individuality. In classical statistical mechanics, he explains, once realistic physical systems such as gases are taken into account and not 'toy systems' such as those composed of idealized coins ${ }^{9}$, it is equally possible to adopt a representation in terms of 'distribution space' (i.e., a description of what

\footnotetext{
${ }^{7}$ Of course, it is possible to claim that this entails that classical particles are nonindividuals too. But individuality is generally taken for granted in the classical domain and so the murkier notion of statistical indistinguishability is normally detached from individuality instead.

${ }^{8}$ See, for example, French and Rickles [2003; 222].

${ }^{9}$ But is a two-fermion system in the singlet state of spin not a realistic system? Such a compound appears analogous to a two-coin classical system. Hence, if we regard the former as a 'serious' physical system, then we should do the same with the latter. More generally, why should certain simple systems not be relevant at the level of the metaphysical reconstruction of reality as Huggett claims?
} 
properties are instantiated where) and of 'phase space' (i.e., the canonical description in terms of individuals with properties). However, only the latter is wedded to haecceitism. Since, in any event, we do not put into question the metaphysical status of classical particles as individuals, it follows from this that no metaphysical consequences should be drawn from our theories, and in particular from the formalism that we have chosen to adopt. This is a general conclusion, Huggett maintains, that applies in the quantum as in the classical case. ${ }^{10}$

Teller [2001] and Gordon [2002], though, contend that the evidence pointed at by Huggett bears witness only to the fact that more 'incomplete' descriptions are sufficient in the classical domain; and that, especially in the quantum case, Huggett's claim that metaphysics is underdetermined by physics is unwarranted. A simple way to spell this out is the following. As was said at the beginning, quantum particles (since they violate the Identity of the Indiscernibles) can be regarded as individuals only if they are attributed primitive thisness. ${ }^{11}$ It follows from this that a connection between individuality and haecceitism must in fact be drawn. For, if particles are individuals because they have primitive thisnesses, it may be the case that two of them differ merely numerically, and so haecceitistic differences must be deemed possible. Therefore, Huggett (correctly) claims that haecceitism is not implied by individuality, but then neglects that only for classical particles is all the information given once their qualities are (and, consequently, haecceitism does not play a role in establishing whether one has individuals or not).

It thus seems that there is indeed a non-negligible difference between the classical and the quantum domain; and that those who want, in spite of this, to maintain that quantum particles are individuals must tackle the evidence directly and not by way of a form of tu quoque reasoning.

\footnotetext{
${ }^{10}$ As regards entropy, Huggett, partly relying on results obtained by van Kampen [1984], contends that there are no observable entropic properties sensitive to a difference between phase space and distribution space. Although what he says in support of this conclusion appears correct, it can be contended that distribution space becomes nevertheless 'more natural' in view of Gibbs' paradox.

${ }^{11}$ I am ignoring here Saunders' abovementioned attempt to show that fermions are individuated purely qualitatively. I also disregard interpretations, alternative to 'orthodox' quantum mechanics, in the context of which the things' individuality coincides with their qualitative uniqueness (for instance, in Bohmian mechanics particles possess exclusive positions sufficient for identifying them unambiguously).
} 
This leads to the consideration of the arguments of the second type, that is, of the arguments that attempt to block the derivation of non-individuality from quantum statistics. With respect to these, the most relevant work is no doubt Belousek's [2000]. Belousek argues that whether quantum systems truly are permutation invariant depends on whether it is correct to assume the Fundamental Postulate of Statistical Mechanics (FPSM) - according to which every distinct equilibrium configuration must be assigned the same statistical weight - in the framework of quantum mechanics. Such an assumption, Belousek argues, is by no means inescapable.

As shown by Tersoff and Bayer [1983], one can derive quantum statistics under a hypothesis of uniformly random a priori distribution of statistical weights over all possible microstates of the system, including permuted ones. Therefore, while given FPSM an assumption of distinguishability (i.e., sensitiveness to permutations) accounts for $\mathrm{MB}$ statistics and one of indistinguishability (i.e., permutation invariance) for quantum statistics, it is possible to obtain BE and FD statistics for distinguishable quantum particles by denying FPSM and postulating a random a priori distribution instead.

FPSM is generally taken to hold because, in absence of any specific information about the system, it seems natural to think that it could be in any of the states available to it with the same probability. FPSM is thus rooted in the Principle of Indifference. However, Belousek contends, the latter is itself object of philosophical debate and is surely far from obviously compelling. Hence, since an assumption of random a priori probabilities may legitimately be regarded as logically weaker than one of equal a priori probabilities, there is room for abandoning FPSM along the lines suggested by Tersoff and Bayer. ${ }^{12}$

Teller and Redhead [2000] raise the objection that to make use of FPSM is objectively preferable whenever there is no maximal ignorance about the system. For, once some information is available, interference terms arise that make uniform priors necessary. An alternative theory based on nonuniform priors, Teller and Redhead explain, should

\footnotetext{
${ }^{12}$ In general, Belousek claims, quantum indistinguishability is conventional in the sense that it depends on a subjective choice between observationally equivalent hypotheses.
} 
satisfactorily deal with all the problems related to well-known no-go theorems. ${ }^{13}$

The foregoing considerations should be sufficient for seeing that the existing proposals for avoiding the move from quantum indistinguishability to quantum non-individuality are not very forceful. In any event, even if one grants that an explanation of the fact that permutations are statistically irrelevant in the quantum domain can be found which is compatible with an ontology of individuals (this is all that the arguments just considered aim to show), the question remains of why non-symmetric states are excluded. ${ }^{14}$

A customary move made by the supporters of the individuality of quantum particles in order to overcome the difficulty with non-symmetric states consists of simply postulating certain primitive and non-further-explicable stateaccessibility restrictions. Systems of indistinguishable particles, on this construal, are never found in non-symmetric states just because this is a fundamental feature of the world. The question arises, however, of whether this is sufficient or not.

Huggett [1995] answers in the affirmative, and suggests that it is explanatory enough to claim that non-symmetric states are simply not in the symmetrised Hilbert space that correctly represents the actual world. Teller [1998], however, objects that this is not satisfactory. He points out that the view of quantum particles as individuals is unable to say why the world is described by a symmetrised Hilbert space while, for example, statistical mechanics can explain why a state of affairs in which a cold cup of tea spontaneously starts to boil is never observed.

Furthermore, Redhead and Teller ([1991] and [1992]) emphasise an additional, independent difficulty: the assumption of alleged basic state-accessibility restrictions on

\footnotetext{
${ }^{13}$ For if we consider the canonical entangled states to actually correspond to distinct equiprobable possibilities we are in fact postulating well-defined separate states for each particle (the differences among which determine the distinctness of the states of the total system). These are, however, exactly the states ruled out by those results going against (local and non-contextual) hidden-variables interpretations of quantum mechanics that constitute part of the 'shared knowledge' about the theory.

${ }^{14}$ The autonomy of this latter issue from that concerning permutations and indistinguishability is not always made crystal-clear in the philosophical literature. It can be easily appreciated, however, by considering the logical possibility of physical systems which, like classical ones, can be in non-symmetric states but whose statistics is, unlike classical statistics, insensitive to permutations.
} 
quantum particles intended as individuals contradicts an essential methodological requirement.

Redhead and Teller's (sensible) working presupposition is that when some meaningful part of a theory does not seem to represent anything, one should elaborate on it further and eventually find the real-world counterpart of the bit of formalism apparently devoid of content. Otherwise, in Redhead's [1975] terminology, one has surplus structure that can never become provided with content and should therefore be eliminated altogether. In the case under consideration, not only are non-symmetric states never experienced; nature would be entirely different if they were realized. Therefore, the meaningfulness of non-symmetric states in quantum theory indeed appears to point to in principle useless surplus structure that one had better get rid of. But this is problematic for those who want to defend the individuality of particles for the following reason. The only way to eliminate the surplus structure represented by the description of non-symmetric states, Redhead and Teller argue, is by opting for a formalism without 'particle labels', i.e., numbers or names appearing in the theory that - putatively - refer to the particles' identities (as in the case of the subscripts in C1-C4 and Q1-Q4 above). The best candidate is the Fock space formalism of quantum field theory. In such a formalism, as in the distribution-space discussed earlier in connection to Huggett's views, only information about 'how many' entities are in a certain state is conveyed and not about 'which entity is what', as labels are dropped. This, however, appears to dispense not only with the labels, but with what they express at the ontological level too: namely, the particles' identities. ${ }^{15}$

This is why there indeed appears to be a tension between the correct methodological approach to surplus structure and the assumption of state-accessibility restrictions on particles as individuals.

Not surprisingly, Huggett does not accept Redhead and Teller's conclusions. First, he claims that in the 'particle-free' quantum field-theoretic formalism states that are superpositions of particle number are possible which appear as

${ }^{15}$ Compare with Huggett's suggestion regarding classical mechanics that the choice of formalism is indifferent and so no metaphysical conclusion should be drawn. Here, the formalism without particle labels becomes instead preferable for methodological reasons and, as a consequence, a hypothesis of non-individuality appears again more plausible. 
surplus structure from the point of view according to which the particle number is constant (in particular, from the perspective of quantum mechanics as a theory about individuals). Hence, says Huggett, the presence of surplus structure is not avoided by shifting to quantum field theory and Fock spaces. In fact, Huggett claims that the whole issue with surplus structure can be reduced to a negligible by-product of our choices of representation which should simply not be given ontological weight. Moreover, Huggett goes on to argue, quantum field theory is empirically equivalent to quantum mechanics, and so the fact that the former makes use of the formalism of Fock spaces cannot entail ontological consequences that are not drawn with respect to the latter as a theory about individuals.

It is undeniable, however, that quantum field theory does not have surplus structure unless it is 'looked at from the outside', as it were, as it does not assume the particle number to be constant; while quantum mechanics appears to have a 'builtin' problem with non-symmetric states as soon as it is assumed that its domain is constituted of well-defined individuals. In addition, it can be legitimately maintained that quantum field theory is in fact not equivalent to quantum mechanics (see, for example, the discussion of Huggett's claim of equivalence in Gordon [2003]) ${ }^{16}$; and that the former lends itself naturally to an ontological interpretation in terms of fields (rather than individual particles), which is not the case for the latter.

More generally, at any rate, one could legitimately ask for a reason to regard particles as individuals in the first place once distribution space is assumed as the correct means to describe systems of many particles (remember that no 'sources' of individuality are available in the quantum domain once haecceitism is ruled out ${ }^{17}$, and that distribution space dispenses with haecceitistic presuppositions).

In the light of this, Redhead and Teller appear to have good reasons to suggest that the mere (unexplained) presence in quantum mechanics of meaningful descriptions of physical states which are never actualised (nor actualisable) is sufficient

\footnotetext{
${ }^{16}$ Gordon emphasises that Fock spaces allow for states that are superpositions of particle number, while many-particle quantum mechanics does not; and that the latter, but not the former, allows for un-symmetrised wave-functions.

${ }^{17}$ Of course, haecceitism is not a source of individuality but rather a consequence of it, at least if the latter is given non-qualitatively. The point is that the fact that haecceitism holds bears witness to an actual fact of - non-qualitatively-determined individuality.
} 
for taking the statistical behaviour of quantum particles as due to their non-individuality.

Given the foregoing discussion, that of the supporters of individuality in quantum mechanics appears to be a rather weak stance, and that quantum particles are non-individuals a natural conclusion to draw. The question is, however, whether such a conclusion is inevitable. The rest of this paper suggests a strategy to answer that it is not, and that it is at least possible to regard quantum particles as individuals, because an explanation of the evidence illustrated above can in fact be provided.

\section{A New Suggestion}

The idea that will be articulated in the rest of the paper is that those who want to defend the position according to which quantum particles are individuals must make a precise ontological claim: they must argue that particles in quantum many-particle systems never possess their state-dependent properties as intrinsic, and that such properties are, instead, always emergent properties of the whole.

In particular, given any many-particle quantum system, they must regard the following as being the case. The total system possesses actual values for its state-dependent properties. The component particles, though, are only related to each other at the level of their dispositions to have specific values for those properties upon measurement. These dispositions, crucially, are not possessed by the particles and are instead 'encoded' in emergent relations holding between them. In a nutshell, the total system exhibits both actual properties and what one may call 'emergent dispositional relations'.

That the statistics is a description of the latter is a natural thing to claim: statistics can be generally intended as a description of possible outcomes of measurements (broadly understood), and it is a widely shared opinion that in quantum mechanics the latter do not uncover already possessed properties but rather determine, in some sense, the possession of actual properties. Here, in particular, I endorse the claim that measurements actualise certain propensities by making emergent dispositional relations 'evolve into' monadic actual (in philosophical vocabulary, 'categorical' as opposed to 'dispositional') properties of their relata. 
Let us get into more detail and see why the present proposal makes a difference with respect to the issue discussed in this paper.

The philosophical literature on emergence is large ${ }^{18}$, but for present purposes it suffices to take an emergent property to be a property $\mathrm{P}$ with the following characteristics:

i) $\mathrm{P}$ is the property of a whole constituted of simpler components;

ii) If $\mathrm{P}$ is a property of the whole composed by parts $a$ and $b$, $P$ is not reducible to the separate properties of $a$ and $b$, but has instead - partly or entirely - 'new content'.

Emergence can thus be regarded here as the denial of mereological supervenience for properties. For a traditional example, think of the property of 'being in mental state $x^{\prime}$. For mind-body dualists, this property is an emergent property of the physical wholes that we call 'persons'. Such a property has the two features above: i) it is attributed to a person as a whole, and a person is an entity with simpler component parts; ii) the contents of one's mental states are not reducible to the properties of one's physical parts. ${ }^{19}$

For emergent relations, the following also holds:

iii) An emergent relation $\mathrm{R}$ is an emergent $n$-adic property of the whole composed of $n$ parts which has parts $x_{1}, x_{2}, \ldots, x_{n}$ as its relata.

So defined, $\mathrm{R}$ is a property exhibited by a whole (call it $\mathbf{S}$ ) which is about the components of $\mathbf{S}$ (as R's relata) but is not reducible to their properties.

In addition, crucially, assume that $\mathrm{R}^{\prime} \mathrm{s}$ 'content' does not include reference to the identities of its relata either. To illustrate this with a useful example, think about two fair coins: of course, since these are classical objects a property of the whole such as, for instance, 'one heads and one tails' is always reducible to two monadic intrinsic properties ('heads' and 'tails') possessed by the coins separately. As a consequence, the property of the

\footnotetext{
${ }^{18}$ For a recent collection of essays on the subject, see Clayton and Davies [2006].

${ }^{19}$ In what follows, obviously enough, only physical components, wholes and properties will be considered.
} 
whole does in fact say which coin is what, and thus includes a reference to specific identities. But if it were possible to have the 'one heads and one tails' property of the two-coin system without having separate properties for the two coins (perhaps because, one could imagine, the former only describes the outcome of a future coin toss), then the property of the whole would be an emergent property that would not say anything about any specific coin. ${ }^{20}$ Note that, in this latter case, switching the coins would not give rise to a new total state: whatever happens to the coins' identities, it is the case that one (without any specification as to which one) will be heads and the other tails. Still, it makes perfect sense to regard the coins as individuals.

This, it is claimed here, is exactly what happens in the case of quantum many-particle systems. For these systems, one only has information about the particles in the form (assuming again two-particle and two-value systems) ' 1 has the same value as 2 for property $\mathrm{P}$, namely, $x^{\prime}$ ' ' 1 has the same value as 2 for property $\mathrm{P}$, namely, $y$ ' or ' 1 has opposite value to 2 for property $\mathrm{P}^{\prime} .{ }^{21}$ According to the present proposal, all these qualitative descriptions, including the first two, correspond to emergent relations of the sort just illustrated. And here too, as for the strange coins above, the descriptions can be taken to be descriptions of individuals.

The idea that all statistically relevant properties of quantum systems are emergent relations is not as 'exotic' as it may seem at first: it essentially consists of an extension to other quantum states of certain widely shared views regarding entangled states. It is commonly claimed that quantum entanglement consists of some form of non-separability, coinciding with the existence of emergent properties that belong to the entire system and not to the system's component particles. Teller [1989] designates as particularism the view that the world is composed of individuals

\footnotetext{
${ }^{20}$ It seems, on the other hand, that emergent relations are not necessarily independent in their 'qualitative content' of the identities of their relata. For instance, to stick to the coin example, one may have a relation saying that coin 1 will be heads and coin 2 will be tails, even though at the time in which the relation holds the coins possess neither a heads or tails value nor a disposition to have one in the future. Alternatively, one may have two coins with well-defined properties, but also additional content in the relation holding between them. For instance, in the form 'coin 1 heads, coin 2 tails and total mass increased by $0.5 \mathrm{MeV}$ with respect to the sum of the coins' separate masses'. Notice, incidentally, the role played by timeasymmetry in defining these relations.

${ }^{21}$ For simplicity, the properties are expressed as if they were categorical and not dispositional here (and below). Strictly speaking, one finds properties such as ' 1 and 2 will (be measured to) have the same value for property $\mathrm{P}$, namely, $x$ ' etc.
} 
possessing non-relational properties, and relations among which supervene on their non-relational properties. He claims that the differences between classical and quantum mechanics are due to the fact that particularism is true of the entities dealt with at the level of the former, but not of those described by the latter. In the quantum domain, Teller argues, one must endorse relational holism: that is, the view that certain properties of the total system are emergent relations entirely independent of the properties of the system's component parts. In particular, Teller considers as a reason to embrace relational holism the failure of outcome-independence in the case of the experimental confirmations of the violation of Bell's inequalities. Relational holism, he holds, allows one to dispense with a tacit assumption of 'ontological locality of values', and consequently renders quantum mechanics compatible with relativity (see Teller [1989; 214-215]). ${ }^{22}$

It can be seen that entangled states, once interpreted from the viewpoint of Teller's relational holism, exhibit emergent relations of the type described above. Consider the singlet state of spin of two fermions. There is a property (the total spin) of a composite system reducible to the properties of the system's parts, which are not in any specific state with respect to their state-dependent properties. The total spin property, however, coincides with a relation describing the future spin-outcomes for the separate fermions in a precise way (as opposite). This latter relation is independent of the fermions' identities, as it does not depict either of them as being in a specific state, nor conveys information as to which fermion will have which value for spin. ${ }^{23}$

Teller's perspective can, therefore, be taken as the starting point here. The crucial addition to it - anticipated above - can be formulated as the suggestion that there is no reason for

\footnotetext{
${ }^{22}$ The idea is that non-locality is avoided in a relational holist context because, according to the latter, in EPR-like settings one does not have a causal relation between two space-like separated events; rather, one has a causal influence on a single entity (the emergent relation), which then 'propagates' to others (its relata) via a causally continuous process (which is immediate in time but also transmitted through a physical continuum - the relation itself - rather than at-a-distance).

${ }^{23}$ There certainly is much to ask about the suggested 'coincidence' between the property of the whole and the relation between the (future) properties of the components. I am assuming here that there exist two distinct properties, one actual and the other dispositional, but perhaps one may put forward a stronger claim of identity and see the two as different 'aspects' of the same property? In any event, nothing hinges on this in the rest of the paper.
} 
saying that the particularist perspective, which is agreed to fail for entangled systems, is valid for non-entangled ones. Indeed, the extension being proposed consists of the claim that quantum relational holism concerns not only entangled but also non-entangled systems; and that, as a consequence, the independence of the entire system's properties (as emergent relations) of the identities of its components (as individuals) generalises to all properties and states. ${ }^{24}$

Eventually getting to the problem being discussed, it can be maintained that the above is all that is needed in order to provide an account of quantum statistics in the context of an ontology of individuals.

First of all, the perspective just envisaged entails that for all many-particle systems and state-dependent properties particle exchanges do not give rise to new arrangements (i.e., the identities of the particles are not statistically relevant) not because particles are not individuals and consequently do not have well-defined identities. Rather, because the particles' identities do not play any role in the determination of the states that are described by the statistics, which are always states that exhibit emergent dispositional relations understood in the precise sense specified in this paper. As in the case of our two imaginary coins, switching the identities of the relata does not affect the qualitative content of the relation that characterises a many-particle quantum system.

A closely related consequence is that one should not expect 'quantum analogues' of classical states such as C4 (that is, nonsymmetric quantum states) to exist, because these would require a property-structure different from the one that - it is being claimed - is exhibited by quantum systems. That is, they would require individual particles that possess well-defined values for their observables separately from each other, which is exactly what is ruled out in the present framework.

Another way to see this point is the following. If relational holism is true of all quantum many-particle systems, it means that the correspondence between states $\mathrm{C} 1$ and $\mathrm{C} 2$ on the one hand and states Q1 and Q2 on the other is only an appearance due to the formalism employed. While the former two effectively are states in which each particle is in a determinate state (that is, possesses a value for the property under

\footnotetext{
${ }^{24}$ Also, but less importantly, the dispositional element emphasised here is not given the same relevance in Teller's work.
} 
consideration as intrinsic), the latter two are instead states in which there is an emergent relation but no determinate states for the relata, exactly in the same way as in the states described by Q3 and Q4. This reading of the situation makes entangled states look immediately much more 'natural' than nonsymmetric ones in the quantum case: for, if Q1 and Q2 were states in which each specific particle possesses a specific property, then Q3 and Q4 would be 'farther removed', as it were, from them than states analogous to C3 and C4, and so there would indeed be a reason to expect the latter to be realized. But if one has instead only emergent relations, then states exhibiting relations attributing equal values to their relata are unsurprisingly 'complemented' by states describing 'opposite value' relations, i.e., by entangled states. As a matter of fact, other kinds of states are necessarily excluded. ${ }^{25}$

What has just been conjectured can hold for all systems, independently of the number of their individual components. To see this, one just needs to conceive of the right emergent relations. For instance, considering three particles and two states, one has $(\mathrm{N}+\mathrm{M}-1)$ ! / N! (M-1)! possible states, namely 4. These are readily described by two 'same value' relations of the sort already encountered, plus two 'different values' relations: 'two particles have the same value for property $\mathrm{P}$, namely, $x$, and one particle has the other value, $y$, for property $\mathrm{P}^{\prime}$; and 'two particles have the same value for property $\mathrm{P}$, namely, $y$, and one particle has the other value, $x$, for property $\mathrm{P}^{\prime} .^{26}$

In fact, if one thinks about it, one can see that the explanation ${ }^{27}$ of quantum statistics suggested here must be deemed satisfactory if an account based on non-individuality is. Because the former differs from the latter only with respect to 'where identity is taken out of the picture', so to speak: property-type rather than property-bearers.

\footnotetext{
${ }^{25}$ Recall the question about non-symmetric states asked in section 1, and the relative footnote regarding the 'tacit assumption' of intrinsic properties for the separate particles.

${ }^{26}$ Again, this neglects the dispositional element for simplicity. Here, the essential fact is that there are only two possible ways for three particles not to have all the same value for a two-valued observable if the relevant information is entirely encoded in emergent (dispositional) relations in the sense assumed here.

${ }^{27}$ It is important to emphasise that here we have an ontological explanation of why there are state-accessibility restrictions that apply to the particles as individuals, not (anymore) an a priori denial of the possibility of certain states being actualised.
} 
In the light of the preceding discussion, questions regarding the 'mysterious non-classicality' of quantum statistics eventually turn out to be less problematic for the supporter of individuality than commonly thought. The specific identities of the separate individual particles, it is possible to claim, are simply irrelevant for the determination of any of the states that the statistics describes, due to the peculiar property-structure exhibited by quantum entities in such states. ${ }^{28}$

\section{Further Remarks}

Let us now consider some possible reactions, and add a few remarks.

i) One may dislike an ontology according to which nonsupervenient relations invariably emerge in quantum manyparticle systems out of particles that possess separate actual (or, 'categorical') properties when they do not belong to the same system. In reply to this sentiment, the following remark can be formulated (again). The fact of emergence being pointed at is something peculiar about the quantum domain in general, and the present proposal simply extends to other systems claims that are already widely accepted for certain physical composites (i.e., entangled systems) under any interpretation of the theory. If an explanation must be sought at all, it must regard the nature of entanglement in general rather than (or at least before) the present suggestion concerning quantum statistics.

ii) One might insist on the presence of in principle meaningless surplus structure in the formalism of quantum mechanics. This response could in that case be given: it can equally be maintained that classical mechanics is inadequate as a description of the objects in its domain because it is possible to describe the latter entities as entangled but entangled states are never realised in the classical world. In general, given any physical theory and its formalism, it appears always possible to 'cook up' some form of surplus structure. In fact, it seems correct to claim that what counts as surplus structure is not immediately determined and ontological presuppositions are fundamental for interpreting the theory. This is essentially the reason why it is contended here that the ontological explanation

\footnotetext{
${ }^{28}$ Notice that such a property-structure might demand in turn an explanation. But this does not involve the particles' identities and, therefore, it does not have to do with (non-)individuality any longer. See point i) in the next section.
} 
provided in this paper succeeds where talk of inexplicable state-accessibility restrictions failed. ${ }^{29}$

iii) Usually, the Eigenstate-Eigenvalue Link is employed when interpreting the quantum formalism. According to it, a physical system actually possesses a specific value for an observable if it is in an eigenstate for that observable corresponding to that value. This licenses inferences such as the following:

$[\operatorname{Prob}($ particle $x$ has property P with value $v)=1] \Rightarrow[($ Particle $x$ actually has property $\mathrm{P}$ with value $v)]$

However, it was denied earlier that in states such as, for instance, Q1 one has two particles each actually possessing a specific value for the given observable as an intrinsic property: the consequent in the above conditional must thus be deemed false. But in such states, the component particles have probability 1 of being detected as having that property (as they are in an eigenstate for that observable): the antecedent is true. Therefore, the Eigenstate-Eigenvalue Link seems to be made invalid by the present proposal.

The response to this is that, according to the ontological hypothesis that was put forward in this paper, one must indeed make an amendment to the Eigenstate-Eigenvalue Link, and regard it as only applying to the total system. According to this interpretation, each separate particle in a many-particle system can be seen as possessing a property as intrinsic only after measurement (when the system will be split into distinct subsystems), even if it has probability 1 of possessing that property before being measured. Before measurement, it is maintained, such a probability only follows from the description of a disposition of the entire system and cannot therefore be regarded as corresponding to an actual property that can be attributed to the specific particle. This modification to the link which is at any rate not an integral part of quantum theory and is modified or even abandoned also in other contexts such as, for instance, modal interpretations of quantum mechanics should appear acceptable. Especially so once one realizes that, although essential from the perspective of one's ontological

\footnotetext{
${ }^{29}$ It is interesting to notice that Huggett [1995] makes the same claim about surplus structure (using the example of the description in the 'language' of classical mechanics of a body moving faster than the speed of light) but by way of conclusion of a paper that attempts to deflate the relevance of metaphysics entirely.
} 
interpretation of quantum theory, such a modification does not make any difference in practice: we can still attribute separate properties to the particles that compose a non-entangled state before measurement exactly because we know from the quantum probabilities that upon measurement they will necessarily possess such properties. Since measurement is the only way to check whether a given quantum particle has a certain (state-dependent) property, to ask whether the particle already has the detected properties before measurement is simply otiose, and no empirical difference can possibly emerge between the two scenarios (i.e., with dispositions encoded in emergent relations and with intrinsic properties - be they dispositional or categorical - respectively).

iv) It could be maintained that the picture delineated in this paper essentially amounts to an endorsement of Bohmian mechanics: the attribution of state-dependent properties to the 'whole system', that is, could be regarded as basically the same as the attribution of them to a 'guiding wave'. There might be something to this criticism, in the sense that the basic idea is in some way inspired by the De Broglie-Bohm view and by the thought that there may be a clear-cut ontological difference between types of quantum properties. ${ }^{30}$ But of course, the important difference exists that no assumption has been made here about uniqueness of positions and initial particle distribution in agreement with $|\Psi|^{2}$, which are two distinguishing features of Bohmian mechanics. Also, crucially, unlike in Bohmian mechanics the notion of collapse is retained in the present framework. Therefore, the analogy is only superficial.

A closely related objection could be that the suggested proposal aims to achieve something which is already obtained by endorsing Bohmian mechanics, and consequently turns out to be superfluous. This criticism, however, can easily turned on its head: the suggested picture of quantum reality, one could argue, achieves some of the allegedly important results of Bohmian mechanics (possibility to describe particles as (quasi)classical objects, reconstruction of the statistics within an

\footnotetext{
${ }^{30}$ As is well-known, Bohmian mechanics takes the particles' state-independent properties and their positions as essential to the particles themselves, while it attributes all the state-dependent properties to a wave component, 'guiding' the particles in space. The exact position occupied by the particles with respect to the wave determines their behaviour including, crucially, the outcomes of measurements of state-dependent properties.
} 
ontology of individuals) without departing from what many see as the correct theory of the quantum world and the correct interpretation of it (namely, the so-called 'orthodox' interpretation of quantum mechanics based on Von Neumann's mathematical formalism and on the notion of collapse of the wave-function). True, if one is happy with Bohmian mechanics, one will presumably find no reason to embrace the perspective defined in this paper. But the present work is primarily directed to those who are, to the contrary, not particularly fascinated by Bohm's theory and would rather stick to standard quantum mechanics (perhaps, provided that the latter could be shown to be consistently interpretable in terms of individuals).

\section{Conclusions}

Contrary to a widespread belief, it can be concluded, it is possible to formulate an explanation of quantum statistics from a perspective according to which quantum particles are individuals. This explanation is based on an extension of property holism (in particular, of Teller's relational holism) to all state-dependent properties of quantum many-particle systems, and an ensuing modification of the EigenstateEigenvalue Link. The resulting ontological picture allows one to claim that quantum statistics is, unlike classical statistics, insensitive to permutations because the latter are not relevant at the level of the property-structure that the statistics describes. And that, in particular, non-symmetric states are not mysteriously unrealisable but rather rendered impossible by the fact that the state-dependent properties of quantum particles in many-particle systems are never possessed by the single particles as intrinsic.

\section{References}

Belousek, D.W., (2000): Statistics, Symmetry, and the Conventionality of Indistinguishability in Quantum Mechanics, in Foundations of Physics, 30, 1-34.

Born, M. (1926): Quantenmechanik der Stobvurgnge, in Zeitschrift für Physik, 38, 803-827.

Clayton, P., and Davies, P. (eds.), (2006): The Re-Emergence of Emergence, Oxford University Press, Oxford.

French, S. and Krause, D., (2006): Identity in Physics: A Historical, Philosophical, and Formal Analysis, Oxford University Press, USA. 
French, S. and Redhead, M., (1988): Quantum Mechanics and the Identity of the Indiscernibles, in British Journal for the Philosophy of Science, 39, 233-246.

Gordon, B.L., (2002): Maxwell-Boltzmann Statistics and the Metaphysics of Modality, in Synthèse, 133, 393-417.

Gordon, B.L., (2003): Ontology Schmontology? Identity, Individuation, and Fock Space, in Philosophy of Science, 70, 5, Proceedings of the 2002 Biennial Meeting of the Philosophy of Science Association. Part I: Contributed Papers, 1343-1356.

Gottesman, D., (2005): Quantum Statistics with Classical Particles, online at arXiv:cond-mat/0511207v2.

Huggett, N., (1995): What are Quanta, and Why Does it Matter?, in Proceedings of the 1994 Biennial Meeting of the Philosophy of Science Association. Part II: Symposia and Invited Papers, 69-76.

Huggett, N., (1997): Identity, Quantum Mechanics and Common Sense, in The Monist, 80, 118-130.

Huggett, N., (1999): Atomic Metaphysics, in The Journal of Philosophy, 96, 5-24.

Redhead, M., (1975): Symmetry in Intertheory Relations, in Synthèse, 32, 77-112.

Redhead, M. and Teller, P., (1991): Particles, Particle Labels, and Quanta. The Toll of Unacknowledged Metaphysics, in Foundations of Physics, 21, 43-62.

Redhead, M. and Teller, P., (1992): Particle-Labels and the Theory of Indistinguishable Particles in Quantum Mechanics, in British Journal for the Philosophy of Science, 43, 201-218.

Saunders, S., (2006): Are Quantum Particles Objects?, in Analysis, 66, 52-63.

Teller, P., (1989): Relativity, Relational Holism, and the Bell Inequalities, in Cushing, J. and McMullin, E., (eds.): Philosophical Consequences of Quantum Theory: Reflections on Bell's Theorem, University of Notre Dame Press, Notre Dame, Indiana, 208-223.

Teller, P., (1998): Quantum Mechanics and Haecceities, in Castellani, E. (ed.), Interpreting Bodies: Classical and Quantum Objects in Modern Physics, Princeton University Press, Princeton, 114-141.

Teller, P., (2001): The Ins and Outs of Counterfactual Switching, in Noûs, 35, 365-393.

Teller, P. and Redhead, M., (2000): Is Indistinguishability in Quantum Mechanics Conventional?, in Foundations of Physics, 30, 951957.

Tersoff, J. and Bayer, D., (1983): Quantum Statistics for Distinguishable Particles, in Physical Review Letters, 50, 553-554.

Van Kampen, N.G., (1984): The Gibbs Paradox, in Parry, E., (ed.): Theoretical Physics, Pergamon, Oxford, 303-312. 